\title{
Frecuencia de SNP en genes candidatos para crecimiento y su efecto en caracteres de peso vivo en ganado para carne de Tamaulipas
}

\author{
Ana María Sifuentes Rincón ${ }^{a^{*}}$ \\ Gaspar Manuel Parra Bracamonte ${ }^{\mathrm{a}}$ \\ Williams Arellano Vera ${ }^{a}$ \\ Pascuala Ambriz Morales ${ }^{\text {a }}$ \\ Antonio Cantú Covarrubias ${ }^{b}$ \\ Víctor Ricardo Moreno Medina ${ }^{a}$
}

a Instituto Politécnico Nacional. Centro de Biotecnología Genómica. Laboratorio de Biotecnología Animal, Blvd. Del Maestro esq. Elías Piña. Col. Narciso Mendoza s/n. Cd. Reynosa, Tamaulipas, México.

b Instituto Nacional de Investigaciones Forestales Agrícolas y Pecuarias, INIFAP, Campo Experimental Las Huastecas. Tamaulipas, México.

*Autor de correspondencia: asifuentes@ipn.mx

\section{Resumen:}

El objetivo del estudio fue analizar las frecuencias alélicas de 28 Polimorfismos de un solo nucleótido (SNP) localizados en genes candidatos para crecimiento en bovinos, así como determinar su efecto sobre rasgos de peso vivo en hatos de las razas Charolais y Simmental. Se tomaron muestras de pelo de 313 animales provenientes de cinco hatos ubicados en cuatro municipios de Tamaulipas y se genotipificaron con la tecnología de Sequenom Mass Array. Todos los marcadores resultaron ser polimórficos en las poblaciones evaluadas y sus frecuencias alélicas fueron significativamente diferentes entre razas $(P<0.05)$. El análisis de asociación determinó que en los animales de la raza Charolais, el marcador PRL+2723 tiene un efecto significativo $(P=0.0350)$ sobre el peso al nacimiento y el marcador localizado en el gen GHR (GHR-6.1), sobre peso al destete $(P=0.0226)$. El GHR-6.1 también se asoció con el peso al año en los animales de la raza Simmental. El marcador LEP-3100 $(P=0.0249)$ también tuvo un efecto significativo sobre el peso al destete en los animales Simmental. El panel probado es 
polimórfico en las dos razas evaluadas y tres de los marcadores tuvieron efecto significativo en los parámetros de peso vivo evaluados, por lo que tienen el potencial de validarse y usarse como una herramienta adicional en la selección y mejoramiento genético del ganado de carne en Tamaulipas.

Palabras clave: Ganado de carne, Eje somatotrópico, Asociación de SNP, Frecuencias alélicas, Peso vivo.

Recibido: 08/11/2017

Aceptado:31/10/2018

En la ganadería bovina, los rasgos económicamente importantes, están determinados por diferentes factores de naturaleza poligénica, por lo que actualmente uno de los grandes retos de la genética animal es determinar la arquitectura genética de estos rasgos, para así incluir la información genómica en los criterios de selección de los animales ${ }^{(1)}$.

Debido a su impacto económico en la producción de ganado, así como la relativa facilidad de registro, el peso al nacer y el peso al destete, han sido dos de los rasgos más estudiados desde la perspectiva de la genética tradicional y actualmente desde un enfoque genómico basado en las aplicaciones de selección asistida por marcadores en el ganado bovino $^{(2,3)}$.

Aunque muchos procesos fisiológicos regulan el crecimiento, el eje somatotrópico, se ha establecido como el principal regulador del desarrollo y crecimiento en bovinos ${ }^{(4,5)}$. La hormona de crecimiento $(\mathrm{GH})$ juega un papel central como un potente regulador de las funciones fisiológicas. La liberación de la GH se estimula por la hormona liberadora de la hormona de crecimiento (GHRH) y se inhibe por la somatostatina. La GH se secreta en altos niveles durante el crecimiento de los animales jóvenes. En los adultos su secreción varia, sin embargo; el estímulo más común de su secreción es una reducción en las concentraciones de glucosa en plasma. Aunque es la principal reguladora del eje, la GH no actúa sola, sino en conjunto con otras hormonas, receptores y proteínas de unión. La GH estimula la liberación de las somatomedinas o factores de crecimiento similar a insulina 1 y 2 (IGF-1 e IGF-2) las cuales también ejercen efecto de regulación por retroalimentación negativa sobre el hipotálamo y la adenohipófisis y tienen influencia sobre el crecimiento. Además de la GH, la prolactina ( $\operatorname{Prl})$ es una hormona polipeptídica también secretada principalmente por la glándula pituitaria, y participa en múltiples procesos endocrinos, la mayoría de sus funciones están relacionadas con lactancia y reproducción en mamíferos a través de vías de señalización que involucran a los factores de transcripción STAT5 ${ }^{(6)}$. 
Existen otras hormonas que, si bien no influyen tanto sobre el crecimiento, si tienen efecto sobre el peso. Por ejemplo, la leptina (LEP) es un regulador de la ingesta de alimentos y el balance energético ${ }^{(7)}$.

En el ganado bovino, los genes de los péptidos y hormonas antes mencionados han sido considerados como candidatos para rasgos productivos, por lo que han sido ampliamente caracterizados y algunos de sus polimorfismos han sido asociados en diferentes razas de bovinos con el peso corporal ${ }^{(5,7,8)}$, el peso de la canal $^{(5,7,9)}$, el rendimiento de la leche y la fertilidad ${ }^{(10,11)}$.

Diferentes estudios realizados en hatos de ganado de la raza Charolais en México han mostrado asociación entre algunos marcadores moleculares localizados en estos genes candidatos $^{(2,12,13)}$. Sin embargo, es muy importante considerar que el patrón de asociación de estos marcadores es ampliamente variable entre las razas y aún más entre las poblaciones; por lo que es necesaria su evaluación antes de implementarse dentro de los esquemas de selección y mejoramiento genético.

Por lo anterior, el objetivo del trabajo fue estimar y comparar las frecuencias alélicas de un panel de marcadores del tipo SNP así como determinar su efecto sobre características de peso vivo en animales de las razas Charolais y Simmental de Tamaulipas.

Se analizaron muestras de 313 animales de hatos ubicados en diferentes municipios del estado de Tamaulipas y obtenidas en los años 2014 y 2015. Las muestras de ganado Charolais $(n=199)$ se obtuvieron de cuatro hatos diferentes localizados en San Fernando (dos hatos), Gustavo Díaz Ordaz y Cd. Victoria; y están representadas por 105 crías nacidas entre 2012 y 2014 (55 hembras y 50 machos), 77 vacas y 17 sementales. Solo en el caso de uno de los hatos de San Fernando se pudo comprobar la relación filial de las crías $(n=13)$ con las vacas y sementales muestreados. En el caso del hato de Díaz Ordaz se pudo comprobar la relación filial de las crías $(n=18)$ con los tres sementales muestreados. En el caso de la raza Simmental $(n=114)$, se incluyeron 32 crías, 70 vacas y 12 sementales provenientes de un solo hato localizado en el municipio de Aldama. Todos los sementales y crías evaluados son animales de registro.

De los cinco hatos evaluados, se obtuvo la información de registro de manejo y productiva disponible para peso al nacimiento (PN), peso al destete ajustado a $205 \mathrm{~d}$ (PD) y peso al año ajustado a 365d (PA), por lo que, en el análisis de asociación, los datos analizados para cada característica varían de los de la muestra total incluida en el estudio.

Con base en la literatura existente, se diseñó un panel de 28 SNP localizados en genes candidatos para crecimiento y peso del animal. Del total de la población, se tomaron muestras de pelo y a partir de éstas, se realizó la tipificación usando el Sistema Sequenom MassARRAY de acuerdo con las especificaciones de la compañía GeneSeek Inc. (Lincoln, NE, USA), 
Una vez obtenidos los genotipos, se estimaron las frecuencias genotípicas y alélicas de los marcadores analizados utilizando módulo Allele Frequency Analysis del programa Cervus $3.0^{(14)}$. Se realizó la prueba exacta de equilibrio Hardy-Weinberg bajo la hipótesis alternativa de déficit de heterocigotos $(P>0.05)$ usando el programa Genepop versión 4.0.10 $0^{(15)}$. En el análisis de diferenciación genética, la hipótesis nula probada fue $\mathrm{Ho}=$ la distribución alélica es idéntica a través de las poblaciones. Para poblaciones, la prueba se realiza por medio de tablas de contingencia por pares de poblaciones y se lleva a cabo una estimación no sesgada del valor de P o Prueba exacta de Fisher para cada $l o c i^{(15)}$.

Con los datos genotípicos y productivos (PN, PD, PA) obtenidos en las razas Charolais y Simmental, se llevó a cabo para cada raza un análisis de asociación. Para aislar todos los efectos no genéticos de los genotípicos, se ajustó en cada caso, el modelo lineal de la siguiente manera:

$\mathrm{Y}_{\mathrm{ijklm}}=\mu+\mathrm{S}_{\mathrm{i}}+\mathrm{A}_{\mathrm{j}}+\mathrm{E}_{\mathrm{k}}+\mathrm{G}_{\mathrm{l}}+\mathrm{H}_{\mathrm{m}}+\beta_{\mathrm{ev}}+\varepsilon_{\mathrm{ijklm}}$,

Donde:

$\mathbf{Y}_{\mathbf{i j k l m}=}$ peso al nacimiento, peso al destete o peso al año;

$\boldsymbol{\mu}=$ media general;

$\mathbf{S}_{\mathbf{i}}=$ efecto fijo del i-ésimo sexo del animal;

$\mathbf{A}_{\mathbf{j}}=$ efecto fijo del j-ésimo año de nacimiento;

$\mathbf{E}_{\mathbf{k}}=$ efecto fijo de la k-ésima época de nacimiento;

$\mathbf{G}=$ efecto fijo del l-ésimo genotipo en el SNP analizado;

$\mathbf{H}_{\mathbf{m}}=$ efecto fijo del m-ésimo hato (solamente para animales Charolais);

$\boldsymbol{\beta}_{\mathbf{e v}}=$ covariable lineal de la edad de la madre,

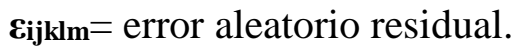

El modelo solo incluyó los factores que fueron significativos en un análisis exploratorio que consideró la evaluación de interacciones de primer orden y el análisis del efecto cuadrático de la edad de la madre. Todos los análisis se realizaron utilizando el procedimiento GLM del paquete estadístico SAS 9.0 (SAS Institute Inc., Cary, NC, USA). Posteriormente, se estimaron las medias de mínimos cuadrados y error estándar de los efectos genotípicos de los SNP y fueron comparadas utilizando el método PDIFF mediante el ajuste Tukey-Kramer en el mismo paquete estadístico.

De acuerdo al análisis de las frecuencias alélicas de los 28 marcadores analizados, todos son polimórficos en las dos poblaciones evaluadas (Cuadro 1). Debido a un déficit de heterocigotos, los marcadores Lep-1457 y Prl-RsaI, mostraron desviación al equilibrio de Hardy-Weinberg, el primero en la raza Simmental $(P<0.0003)$ y el segundo en la Charolais $(P<0.0001)$. En ambos casos, el tamaño de la muestra podría ser la principal explicación para la desviación observada, otras causas probables son el nivel de endogamia debido a los pocos sementales usados en los hatos. 
Cuadro 1: Frecuencias alélicas del panel de 28 SNP evaluados en las razas Charolais y Simmental

\begin{tabular}{|c|c|c|c|c|c|c|c|c|}
\hline \multirow{2}{*}{ SNP } & \multicolumn{4}{|c|}{ Charolais } & \multicolumn{4}{|c|}{ Simmental } \\
\hline & $\mathbf{A}$ & $\mathbf{C}$ & $\mathbf{G}$ & $\mathbf{T}$ & $\mathbf{A}$ & $\mathbf{C}$ & $\mathbf{G}$ & $\mathbf{T}$ \\
\hline GHR-1.1 & 0.9020 & & 0.0980 & & 0.7479 & & 0.2521 & \\
\hline GHR-1.4 & 0.5758 & & 0.4242 & & 0.4871 & & 0.5129 & \\
\hline GHR-2.6 & & 0.1106 & & 0.8894 & & 0.0304 & & 0.9696 \\
\hline GHR-2.6 & 0.1106 & & 0.8894 & & 0.0302 & & 0.9698 & \\
\hline GHR-4. & & & 0.4444 & 0.5556 & & & 0.3803 & 0.6197 \\
\hline GHR-6.1 & 0.2186 & & 0.7814 & & 0.4786 & & 0.5214 & \\
\hline GHR-A536 & 0.0354 & & 0.9646 & & 0.0302 & & 0.9698 & \\
\hline GHR-F279 & 0.0528 & & & 0.9472 & 0.1043 & & & 0.8957 \\
\hline GHR-H54 & & 0.6332 & & 0.3668 & & 0.7241 & & 0.2759 \\
\hline GHR-N528 & 0.3995 & 0.6005 & & & 0.3319 & & 0.6681 & \\
\hline GHR-S555 & 0.5452 & & 0.4548 & & 0.6034 & & 0.3966 & \\
\hline GHRH+2279 & & 0.3543 & & 0.6457 & & 0.2328 & & 0.7672 \\
\hline GHRH-2298 & 0.3819 & 0.6181 & & & 0.4655 & 0.5345 & & \\
\hline GHRH-4241 & 0.3593 & & & 0.6407 & 0.3922 & & & 0.6078 \\
\hline IGF1/Sna BI & & 0.6633 & & 0.3367 & & 0.5690 & & 0.4310 \\
\hline LEP-1180 & & 0.6106 & & 0.3894 & & 0.7802 & & 0.2198 \\
\hline LEP-1457 & 0.5941 & & 0.4059 & & 0.6173 & & 0.3827 & \\
\hline LEP-3100 & & 0.7437 & & 0.2563 & & 0.8233 & & 0.1767 \\
\hline LEP-3157 & 0.9898 & & 0.0102 & & 0.9957 & & 0.0043 & \\
\hline LEP-3257 & & 0.1658 & & 0.8342 & & 0.2586 & & 0.7414 \\
\hline LEP-3272 & & 0.1658 & & 0.8342 & & 0.2586 & & 0.7414 \\
\hline LEP-978C & & 0.4975 & & 0.5025 & & 0.7802 & & 0.2198 \\
\hline LEPY7FA & 0.8065 & & & 0.1935 & 0.9914 & & & 0.0086 \\
\hline PRL/Rsa I & 0.3081 & & 0.6919 & & 0.1121 & & 0.8879 & \\
\hline PRL2723 & & 0.2663 & & 0.7337 & & 0.2716 & & 0.7284 \\
\hline $\begin{array}{l}\text { STAT1- } \\
\text { C213 }\end{array}$ & & 0.9121 & & 0.0879 & & 0.9430 & & 0.0570 \\
\hline $\begin{array}{l}\text { STAT5A- } \\
12735\end{array}$ & & 0.9564 & & 0.0436 & & 0.8190 & & 0.1810 \\
\hline bGH/Alu I & & 0.7828 & 0.2172 & & & 0.7888 & 0.2112 & \\
\hline
\end{tabular}


La comparación de las frecuencias alélicas permitió observar que en 25 marcadores éstas son significativamente diferentes entre las poblaciones evaluadas $(P<0.001)$. Solo en el caso de los marcadores GHR-1.1 ( $P>0.389)$, Lep-3157(P>0.516) y GH/Alu I $(P>0.649)$, no se encontraron diferencias significativas entre las dos poblaciones comparadas.

Aunque todos los marcadores evaluados en este estudio tienen el potencial de asociación con rasgos productivos, se destacan las frecuencias de los marcadores GH/Alu I., IGF1-Sna BI y Lep-1180, debido a que se han asociado a rasgos productivos como pesos del animal, calidad de canal y de la carne en poblaciones de ganado de diferentes razas tanto Bos taurus taurus como Bos taurus indicus ${ }^{(16-20)}$.

La transversión de $\mathrm{C} / \mathrm{G}$ en la posición nucleotídica 2141 del exón 5 del gen GH (marcador GH/Alu I), produce un cambio de valina (alelo G) por leucina (alelo C) en la posición aminoacídica 127 del gen. En trabajos previos, se ha demostrado que animales portadores del alelo favorable $\mathrm{C}$ suelen estar relacionados con un mayor marmoleo $\mathrm{y}$ peso de la canal ${ }^{(21,22)}$. Para este marcador, las razas evaluadas presentaron una mayor frecuencia del alelo favorable; este resultado también se presentó para el marcador IGF1/Sna B1, siendo este, una transición T/C localizada en la posición -472 de la región 5' no codificante del gen IGF1. Algunos estudios han encontrado que, para este marcador, los animales portadores del alelo favorable $\mathrm{C}$, presentan asociación con mayores ganancias de peso al destete que los portadores del alelo $\mathrm{T}^{(8,18)}$.

El marcador Lep-1180, es una transición no sinónima de citosina (C) por timina (T) localizada en el exón 2 del gen de la leptina, que produce un cambio aminoacídico de arginina a cisteína ${ }^{(23)}$. Algunos estudios han encontrado que los alelos de este marcador se encuentran asociados con el contenido de grasa dorsal y con la suavidad de la carne $^{(16,23)}$.

Las poblaciones incluidas en este estudio mostraron una menor frecuencia del alelo $\mathrm{T}$ considerado como el alelo favorable para las diferentes características de la canal antes mencionadas. Este resultado puede ser un reflejo de que la presión de selección en las razas para carne en México se ha inclinado por los rasgos que son fáciles de medir y registrar como lo es el peso vivo, más que para aquellos asociados con los rasgos de calidad de la carne ${ }^{(2,12)}$.

En la población de la raza Charolais, el análisis de asociación indicó que el marcador PRL+2723 ubicado en el gen Prl tiene un efecto significativo $(P=0.0350)$ sobre el peso al nacimiento (Cuadro 2). La media del peso en los animales con el genotipo homocigoto $\mathrm{CC}(44.69 \mathrm{~kg})$ fue $5.66 \mathrm{~kg}$ mayor que el genotipo homocigoto TT y 6.43 $\mathrm{Kg}$ que el heterocigoto $\mathrm{CT}$. 
Cuadro 2: Marcadores con efecto significativo sobre variables de peso de las razas Charolais y Simmental

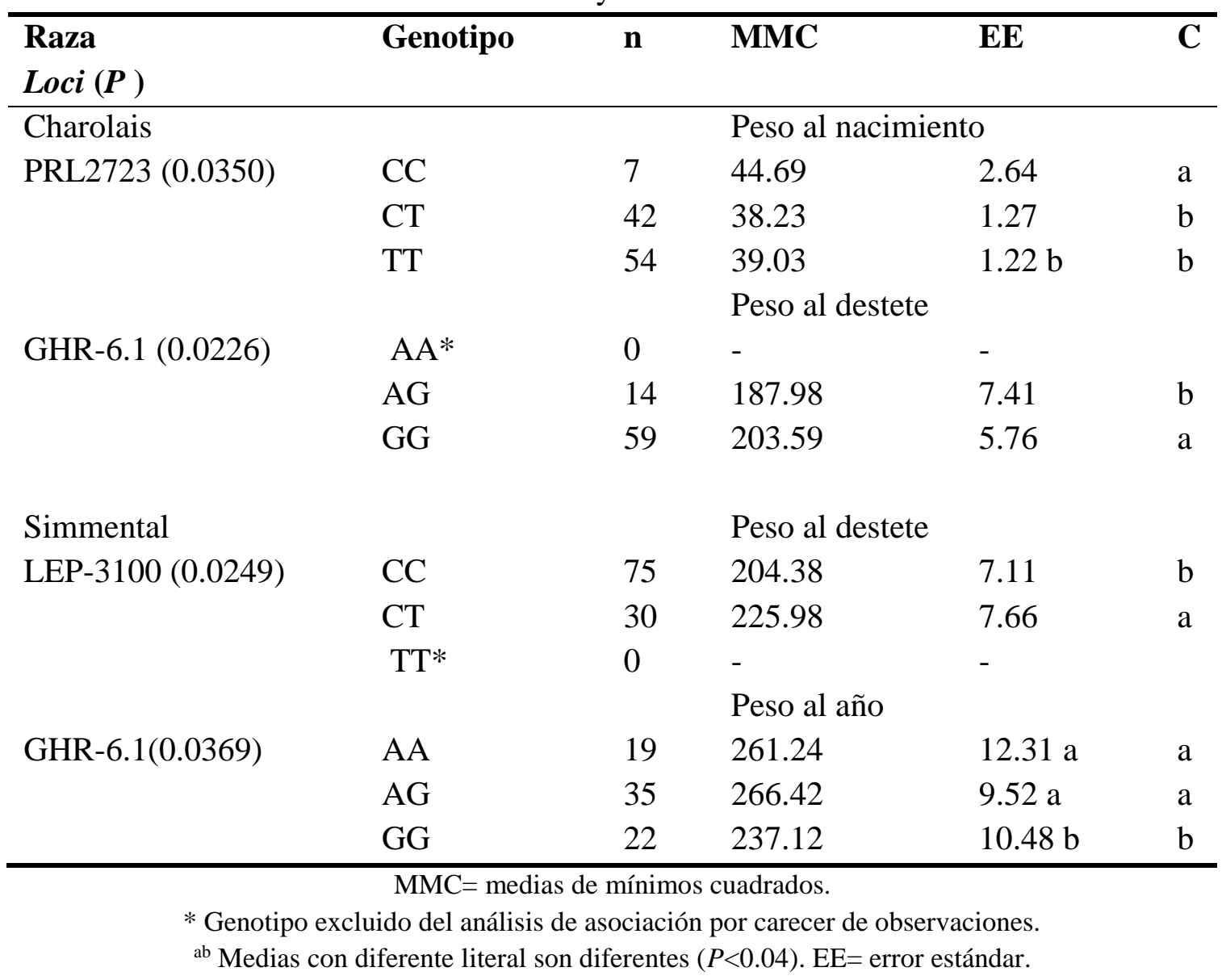

Debido a su papel crucial en el desarrollo de la glándula mamaria, lactogénesis y regulación de la expresión de importantes genes involucrados en la producción de leche, el gen de la prolactina se ha posicionado como un fuerte candidato para la selección asistida por marcadores. En este gen se han identificado algunos SNP con asociación a diferentes rasgos, en particular el SNP PRL-2723, se encuentra localizado en el intrón 1 del gen y aunque ha sido incluido en algunos trabajos de la literatura, hasta la fecha no se ha encontrado efecto positivo o negativo de esta sustitución alélica. En el caso del ganado de carne, cambios en la producción y composición de la leche podrían verse reflejados en la cría ${ }^{(24)}$.

Un resultado relevante del análisis de asociación fue el encontrar que el marcador GHR6.1, tuvo efecto sobre dos parámetros de peso vivo en las dos razas evaluadas. En la raza Charolais, el marcador mostró efecto significativo sobre el peso al destete. La media del peso en los animales con el genotipo homocigoto GG $(203.59 \mathrm{~kg})$ fue 15.61 $\mathrm{kg}$ mayor que el genotipo heterocigoto $\mathrm{AG}(187.98 \mathrm{Kg})$. Debido a la baja frecuencia de portadores $(\mathrm{n}=0)$, el genotipo AA fue excluido del análisis de asociación. En la población de la raza Simmental, el marcador GHR-6.1 se asoció con el peso al año. Los animales con genotipo AA y AG fueron más pesados al año que los animales con genotipo GG (Cuadro 2). 
Como muchos otros genes del eje somatotrópico, polimorfismos localizados en este gen, se han asociado a diferentes rasgos productivos principalmente en ganado lechero. En ganado de carne mexicano, este mismo marcador (GHR6.1) explicó aproximadamente $9 \%$ de la varianza genética $(P=0.0877)$ del peso al nacimiento, con un $\alpha_{\mathrm{G}>\mathrm{A}}=0.509^{(12)}$; los resultados descritos se obtuvieron en una población constituida por hatos de ganado Charolais localizados en Nuevo León y Sonora ${ }^{(12)}$. Una práctica común de los productores de Charolais en México, es recurrir a las ganaderías de Nuevo León para la compra de material genético para el mejoramiento genético de sus hatos, por lo que evaluar el efecto de estos marcadores sobre los diferentes fenotipos de peso vivo, permitirán validar este locus como candidato para asistir las estrategias de selección de la raza Charolais en México. Este sería el mismo caso de la raza Simmental, ya que en la población estudiada también se encontró asociación del marcador GHR-6.1 con el peso al año.

En la población Simmental, también se encontró efecto del marcador LEP-3100 con una asociación importante en el peso al destete. La media de los genotipos CT $(225.98 \mathrm{~kg})$ fue de $21.6 \mathrm{~kg}$ mayor que él genotipo homocigoto CC. Debido al bajo número de portadores $(n=4)$, el genotipo TT fue excluido del análisis de asociación.

El gen leptina, se considera como uno de los candidatos biológicos más importantes para estudiar el engrasamiento corporal en animales y humanos ${ }^{(19)}$. La leptina es una de las muchas hormonas que participan en la regulación del metabolismo intermediario a través de mecanismos efectores que involucran factores de crecimiento como IGF-1. En ganado bovino, el gen ha sido ampliamente estudiado y se han descrito diferentes polimorfismos que se han asociado a rasgos productivos que involucran el metabolismo de energía, adiposidad y reproducción. El gen también se ha asociado a la regulación del peso corporal, mediando el metabolismo de ganancia de peso $^{(19)}$.

El polimorfismo LEP-3100 es una transición en el exón 3 del gen que causa un cambio aminoacídico de Ala>Val en la posición 80. Este polimorfismo se ha asociado positivamente a la composición de ácidos grasos en la carne, particularmente el alelo $\mathrm{C}$ de este polimorfismo se asoció positivamente con el contenido del ácido graso C14:1 ${ }^{(20)}$. Este es el primer estudio en el que se prueba la asociación de este marcador con el peso al destete el cual es considerado un indicador importante de la ganancia de peso y productividad en la producción de carne dentro de esta raza.

Se encontró que los 28 SNP localizados en genes candidatos para crecimiento son polimórficos en las poblaciones analizadas y muestran frecuencias alélicas significativamente diferentes entre las dos razas evaluadas. El análisis de asociación permitió identificar en las dos razas estudiadas, tres marcadores con efecto significativo en los parámetros de peso vivo evaluados. 


\section{Agradecimientos}

Se agradece el apoyo otorgado por el CONACYT (proyecto clave 294826) y al Instituto Politécnico Nacional (proyecto SIP-IPN No. 20180727) y a los productores ganaderos de Tamaulipas, por su participación y consentimiento para la toma de muestras y uso de la información.

\section{Literatura citada:}

1.- Glazier AM, Nadeau JH. Aitman TJ. Finding genes that underlie complex traits. Science 2002;(298):2345-2349.

2.- Jahuey-Martínez FJ, Parra-Bracamonte GM, Sifuentes-Rincón AM, MartínezGonzález JC, Gondro C, García-Pérez CA, et al. Genome-wide association analysis of growth traits in Charolais beef cattle. J Anim Sci 2016;94(11):4570-4582.

3.- Takasuga A. PLAG1 and NCAPG-LCORL in livestock. J Animal Sci 2016;(87):159-167.

4.-Luna-Nevarez P, Rincon G, Medrano JF, Riley DG, Chase CC, Coleman SW, et al. Single-nucleotide polymorphisms in the growth hormone-insulin-like growth factor axis in straight bred and crossbred Angus, Brahman, and Romosinuano heifers: Population genetic analyses and association of genotypes with reproductive phenotypes. J Anim Sci 2011;(89):926-934.

5.- Sherman EL, Nkrumah JD, Murdoch BM, Li C, Wang Z, Fu A, et al. Polymorphisms and haplotypes in the bovine neuropeptide $\mathrm{Y}$, growth hormone receptor, ghrelin, insulin-like growth factor 2, and uncoupling proteins 2 and 3 genes and their associations with measures of growth, performance, feed efficiency, and carcass merit in beef cattle. J Anim Sci 2008;(86):1-16.

6.- Lu A, Hu X, Chen H, Jiang J, Zhang C, Xu H, et al. Single nucleotide polymorphisms in bovine PRL gene and their associations with milk production traits in Chinese Holsteins. Mol Biol Rep 2010;(37):547-551.

7.- da Silva RCG, Ferraz JBS, Meirelles FV, Eler JP, Balieiro JCC, Cucco DC, et al. Association of single nucleotide polymorphisms in the bovine leptin and leptin receptor genes with growth and ultrasound carcass traits in Nellore cattle. Genet Mol Res 2012;11(4):3721-3728.

8.- De la Rosa-Reyna XF, Montoya-Martínez HM, Castrellón VV, Sifuentes-Rincón AM, Parra-Bracamonte GM, Arellano-Vera W. Polymorphisms in the IGF1/Sna B1 gene and their effect on growth traits in Mexican beef cattle. Genet Mol Res 2010;9(2):875-883. 
9.- Cheong HS, Yoon DH, Park BL, Kim LH, Bae JS, Namgoong S, et al. A single nucleotide polymorphism in CAPN1 associated with marbling score in Korean cattle. BMC Genet 2008;(9):33-10.

10.- Oikonomou G, Michailidis G, Kougioumtzis A, Avdi M, Banos G. Effect of polymorphisms at the STAT5A and FGF2 gene loci on reproduction, milk yield and lameness of Holstein cows. Res Vet Sci 2011;91(2):235-239.

11.- Ruprechter G, Carriquiry M, Ramos JM, Pereira I, Ana M. Metabolic and endocrine profiles and reproductive parameters in dairy cows under grazing conditions: effect of polymorphisms in somatotropic axis genes. Acta Vet Scand 2011;53:35.

12.- Parra GM, Lopez N, Sifuentes AM, Morris S, Lopez LA, Meza LA. Single and composite influence of growth-related candidate gene polymorphisms on additive genetic variation of birth weight in Charolais beef cattle. Trop Anim Health Prod 2014;(46):509-512.

13.- Paredes-Sánchez FA, Sifuentes-Rincón AM, Segura-Cabrera A, García-Pérez CA, Parra-Bracamonte GM. Ambriz-Morales P. Associations of SNP located at candidate genes to bovine growth traits prioritized with an interaction networks construction approach. BMC Genet 2015;16(91):1-12.

14.- Kalinowski ST, Taper ML, Marshall TC. Revising how the computer program CERVUS accommodates genotyping error increases success in paternity assignment. Mol Ecol 2007;(6):1099-1106.

15.- Rousset F. Genepop'007: a complete reimplementation of the Genepop software for Windows and Linux. Mol Ecol Resour 2008;8:103-106.

16.-Schenkel FS, Miller SP, Yee X, Moore SS, Nkrumah JD, Li C, et al. Association of single nucleotide polymorphisms in the leptin gene with carcass and meat quality traits of beef cattle. J Anim Sci 2005;(83):2009-2020.

17.- Unanian M, Barreto C, Ribeiro-de Freitas A, Torres C, Josahkian, LA. Associations between growth hormone gene polymorphism and weight traits in Nellore bovines. Rev Bras Zootec 2000;(29):1380-1386.

18.- Siadkowska E, Zwierzchowski L, Oprzadek J y Strzalkowska N. Effect of polymorphism in IGF-1 gene on production traits in Polish Holstein-Friesian cattle. Anim Sci Pap Rep 2006;(3):225-237.

19.- Matos-Almeida S, Amazonas E, TerraI G, Pereira J, Bayard DP, de Azevedo T. Association between molecular markers linked to the Leptin gene and weight gain in postpartum beef cows. Cienc Rural 2007;37(1):206-211. 
20.- Orrù L, Cifuni GF, Piasentier E, Corazzib M, Bovolenta S, Moioli B. Association analyses of single nucleotide polymorphisms in the LEP and SCD1 genes on the fatty acid profile of muscle fat in Simmental bulls. Meat Sci 2011;(87):344-348.

21.- Tatsuda K, Oka A, Iwamoto E, Kuroda Y, Takeshita H, Kataoka H, et al. Relationship of the bovine growth hormone gene to carcass traits in Japanese black cattle. J Anim Breed Genet 2008;(125):45-49.

22. Grochowska R, Lundén A, Zwierzchowski L, Snochowski M ,Oprzadek J. Association between gene polymorphism of growth hormone and carcass traits in dairy bulls. Anim Sci 2001;(72):441-447.

23.- Buchanan FC, Fitzsimmons CJ, Van Kessel AG, Thue TD, Winkelman-Sim DC, Schmutz SM. Association of a missense mutation in the bovine leptin gene with carcass fat content and leptin mRNA levels. Genet Sci Evol 2002;(34):105-116.

24.- Pacheco-Contreras VI, Parra-Bracamonte GM, López-Bustamante LA, MorenoMedina VR, Sifuentes-Rincón AM. Milk composition and its relationship with weaning weight in Charolais cattle. Rev Bras Zootec 2015;(44):207-212. 